\title{
TADEL VERPFLICHTET. INDIZIERUNG VON MUSIK UND IHRE WIRKUNG
}

\section{Michael Custodis}

Als im Winter 2005/06 französische Vorstädte von gewalttätigen Unruhen erschüttert wurden und man sich umgehend auf die Suche nach Erklärungen und Sündenböcken machte, waren Musiker einmal mehr Thema in politischen Nachrichtensendungen und Magazinen. Französische Rapper wie Monsieur R und Salif dienten in der öffentlichen Wahrnehmung als Stellvertreter einer Entwicklung, die als Besorgnis erregende Mischung aus Jugendkultur, Arbeitslosigkeit, Migrantenbiografien und sozialer Unterschicht beschrieben wurde. Verschiedene Vertreter von Presse und Politik schlossen daraus auf eine direkte Verbindung zwischen Jugendgewalt und HipHop. ${ }^{1}$ Einigen Musikern unterstellte beispielsweise der damalige Innenminister und heutige Staatspräsident Nicolas Sarkozy staatsgefährdende, rassistische und gewalttätige Tendenzen und kündigte medienwirksam gerichtliche Schritte an (Müller/Wunder 2005). Auch in Deutschland kamen ähnliche Diskussionen über den Zusammenhang von Musik und Gewalt wieder einmal auf und machten Rapper wie die Berliner Sido und Bushido zu gefragten Interviewpartnern.

Verdichtet man in diesem Beispiel die Ansichten der öffentlichen Funktionsträger auf ihr Grundmuster, so wird die von der Mehrheitsgesellschaft ausgeübte Deutungshoheit über Normen, Regeln und Werte in dem Augenblick zum Konfliktfeld, wenn Subkulturen autonome soziokulturelle Räume beanspruchen und die ihnen gesetzten Regeln und Grenzen in Frage stellen. Gerade die Auseinandersetzung von Jugendlichen mit der Erwachsenenwelt, der sie mit jedem Lebensjahr und wachsenden Verpflichtungen und Zwängen stetig näher rücken, funktioniert sehr stark über Abgrenzungsmechanismen. Bezieht man diese allgemeine Skizze auf musikalische Phänomene der Jugendkultur, deutet sich bereits die Spannung zwischen der Selbstinszenierung jugendlicher Subkulturen und der staatlichen Kontrolle von Produktio-

1 Dieses Verdikt begegnet in regelmäßigen Abständen immer wieder im Schrifttum zur Popularmusik (vgl. als aktuelle Variation des Themas Miehling 2006). 
nen ihrer musikalischen Trendsetter an, die im Folgenden anhand zweier Beispiele näher bestimmt werden soll. Die hierfür ausgewählten Genres (Punk und HipHop) sind vor allem auch medial sehr präsente Massenphänomene, die ihre Legitimation und ihre Motivation zwar aus dem Rückhalt ihrer spezifisch abgegrenzten soziokulturellen Umgebung ziehen, zugleich aber auf Schichten übergreifende Beachtung setzen. Diese Beachtung ist nicht immer mit Akzeptanz zu verwechseln, sondern wird oftmals über Provokationen und Tabubrüche erreicht.

So, wie die Verletzung von Normen mit Sanktionen verbunden ist und Instanzen über die Einhaltung der allgemeingültigen Regeln wachen, gibt es auch im Bereich der Jugendkultur eine entsprechende Institution, die angezeigten Streitfällen nachzugehen hat: die Bundesprüfstelle für jugendgefährdende Medien (BPjM). ${ }^{2}$ Die folgende Darstellung zweier Fallbeispiele basiert auf den normalerweise unveröffentlichten Abschlussgutachten der zuständigen Kommissionen. ${ }^{3}$ Dank dieses außergewöhnlichen Materials lassen sich die Sichtweisen der jugendlichen Subkulturen einmal aus der Gegenperspektive einer staatlichen Behörde betrachten, die vor der schwierigen Aufgabe steht, in immer neuen Einzelfallentscheidungen die Freiheit der Kunst gegen den Jugendschutz abzuwägen. Nicht ohne Grund untersteht die Bundesprüfstelle dem Bundesfamilienministerium und bindet in ihren Entscheidungsgremien öffentliche, politische, klerikale und künstlerische

2 Auf Antrag von Jugendämtern, der Kommission für Jugendmedienschutz und dem Bundesministerium für Familie, Senioren, Frauen und Jugend sowie auf Anregung von Polizeidienststellen, Schulen und anderen öffentlichen Anregungsberechtigten überprüft die 1954 gegründete Bundesprüfstelle eine Vielzahl von Gegenständen wie Comics, Buchtitel, Broschüren, Zeitschriften, Tonträger und Internetseiten auf jugendgefährdende Inhalte; bis heute wurden etwa 15000 Verbote ausgesprochen. Die Prüfstelle ist nicht für Tageszeitungen, Fernsehen und Hörfunk zuständig, auch die vom Gremium der »freiwilligen Selbstkontrolle« (FSK) vorgenommenen Altersfreigaben von Kino- und Fernsehfilmen fallen nicht in ihren Zuständigkeitsbereich. Die von der BPjM beschlossenen Verbote bedeuten in der Praxis, dass alle beanstandeten Medien nicht beworben und nicht an Kinder und Jugendliche verkauft, ihnen überlassen oder sonst wie zugänglich gemacht werden dürfen. Indizierte Gegenstände dürfen nur unter der Ladentheke in Geschäften oder im Versandhandel angeboten werden, wenn gewährleistet werden kann, dass sie nicht in die Hände von Kunden unter 18 Jahren gelangen. In Fällen politischer oder anderer strafrechtlich relevanter Inhalte kommt eine Indizierung einem vollständigen Verbot gleich, da die üblicherweise weit auszulegende, allenfalls durch den Jugendschutz eingeschränkte Meinungs- und Kunstfreiheit dann nicht gilt.

3 Die getroffenen Entscheidungen werden im Bundesanzeiger monatlich bekannt gemacht, der vom Bundesministerium der Justiz herausgegeben wird (BPjM 2008). An dieser Stelle sei Martina Hannak-Mayer, Referentin der Bundesprüfstelle für jugendgefährdende Medien in Bonn, herzlich für die Überlassung der zitierten Indizierungsgutachten gedankt. 
Interessengruppen ein, ${ }^{4}$ um den unterschiedlichen gesellschaftlichen Meinungen Rechnung zu tragen, eine politische Instrumentalisierung der Behörde zu verhindern und staatliche Zensur auszuschließen (Carus/Hannak-Mayer/ Kortländer 2006: 6). ${ }^{5}$

Bezeichnenderweise war in den beiden hier diskutierten Fällen weder im kleineren, mit drei Mitgliedern besetzten, noch im zwölf Personen umfassenden Entscheidungsgremium ein Musikspezialist involviert. Dieser Umstand beeinflusste zum einen direkt die Art und Weise, wie in den Verfahren über die Inszenierung musikalischer Leitfiguren und prestigeträchtiger Gegenstände sowie über die Idealisierung provokanter Stereotype diskutiert wurde. ${ }^{6}$ Zum anderen erzeugten die ausgesprochenen Indizierungen, die dem Schutz von Kindern und Jugendlichen vor schädlichen Einflüssen dienen sollten, die unerwünschte Nebenwirkung, die beanstandeten Musikstücke in der jeweiligen Szene zu nobilitieren. Dieser Reibungspunkt zwischen einer Behörde auf der einen Seite, die zur Objektivität angehalten ist, um den Spielraum von Kunst in einer Demokratie zu bestimmen, und den kreativen Akteuren der Jugendszenen auf der Gegenseite, die ihren Erfolg auch am Widerstand messen, der ihnen von der Erwachsenenwelt entgegengebracht wird, erweist sich dabei als Schnittstelle von verschiedenartigen Kanonisierungsprozessen.

Kanonisierungen sind ihrer Struktur nach normierende Bündelungen von ausgewählten Eigenschaften, Handlungsweisen oder Wissensgegenständen, um die Überfülle von verfügbaren Alternativen zu begrenzen und der Pro-

4 Die Mitglieder des so genannten 12er-Gremiums, das über Indizierungen entscheidet, stammen aus den Bereichen Kunst, Literatur, Buchhandel und Verlegerschaft, sind ferner Anbieter von Bildträgern und von Telemedien, Träger der freien und der öffentlichen Jugendhilfe, Vertreter der Lehrerschaft sowie von Kirchen und anderen Religionsgemeinschaften, die den Status einer Körperschaft des öffentlichen Rechts besitzen.

5 Vgl. als Parallelfall mit großer Breitenwirkung die von Theodore Gracyk (1996: 125f.) dokumentierten, von Al Gore 1985 initiierten Anhörungen von Rock- und Heavy Metal-Musikern vor dem US-amerikanischen Kongress sowie das von seiner Frau Tipper Gore gegründete Parent's Music Resource Center (PMRC) (Gracyk 1996: 125f.). Mithilfe guter politischer Kontakte bemühte sich das PMRC, direkt juristisch als auch indirekt über Bücher und Einflussnahme auf die Schulpolitik insbesondere gegen Rap und Heavy Metal vorzugehen, und bewirkte bspw., dass alle seither beanstandeten Musikproduktionen mit dem bekannten Aufkleber »parental advisory explicit lyrics « gekennzeichnet werden müssen (Chastagner 1999).

6 Ein weiteres Beispiel findet sich im weiter unten besprochenen Verfahren gegen das Berliner Aggro-Label. So wurde die vom Label herangezogene Expertise zum sozialen Kontext von US-amerikanischem Rap nicht von einem Musikfachmann, sondern von einem Kunsthistoriker und Kultursoziologen erstellt und bezog sich ausschließlich auf typische Inhalte von Songtexten. 
duktion neuer Inhalte als konsensfähiger Maßstab zu dienen. Bezieht man diesen Mechanismus auf die Indizierungsverfahren, tritt der Widerstreit der Interessen noch deutlicher hervor: Beide Seiten - die Mehrheitsgesellschaft, vertreten durch die staatliche Behörde, sowie die subkulturellen Strömungen der Jugendszenen (repräsentiert durch anerkannte Meinungsführer), die solche Eingriffe als Bevormundung empfinden und gar nicht beschützt werden wollen - sammeln entlang der bestehenden Grenzlinie zwischen Kunstfreiheit und Jugendschutz Erfahrungen im Umgang miteinander. Die erlassenen behördlichen Beschränkungen umstrittener Produktionen wirken dabei nicht eindimensional schützend auf die jugendliche Zielgruppe und präventiv mäßigend auf die von innen favorisierten Künstler ein. Vielmehr reichen die Folgen von Indizierungen zu mindestens gleichen Teilen bis zu den begrenzenden Instanzen zurück, wenn Musiker ihre folgenden Produktionen bewusst provokativ konzipieren, um die behördliche Beschränkung zum Aufbau eines Images als offiziell »anerkannte« Bad Boys zu funktionalisieren. Entsprechend der zuvor beschriebenen Struktur kanonisierter Verhaltensmuster und Wertesysteme - so die These der folgenden Ausführungen - werden die Regeln der Bundesprüfstelle innerhalb einzelner Jugendszenen daher genau umgekehrt interpretiert und in Form von besonders kritisierten Haltungen, Formulierungen oder Verhaltensweisen im Sinne eines Negativ-Maßstabs kanonisiert.

\section{Punk - Die Ärzte}

Die Arbeitsweise der Bundesprüfstelle lässt sich am Beispiel der Berliner Punkrockband Die Ärzte besonders gut beleuchten, da die Aufhebung der 1987 erlassenen Indizierung der Titel »Claudia hat 'nen Schäferhund« und "Schlaflied« im Jahr 2004 in der Geschichte der BPjM nahezu singulär ist. Ein Vierteljahrhundert später attestierte man der Gruppe nun humorige, satirische und ironische Motive.

Bis auf eindeutig identifizierbare Melodien wie bspw. das »Horst-WesselLied« geben üblicherweise die Texte Anlass zur Indizierung von Musikstücken, und - wenig überraschend - entstammt keiner der derzeit indizierten Titel der Kunstmusik. Ein Verstoß gegen die ausgesprochenen Verbote kann strafrechtliche Konsequenzen nach sich ziehen, wie im Fall der Ärzte nach einem Konzert am 22. Juni 1988 in Kleve. Bei dieser Gelegenheit hatten die Musiker ihr Publikum, bevor sie eine Instrumentalversion des indizierten Songs »Geschwisterliebe « spielten, überdeutlich dazu aufgefordert, auf keinen Fall den Text mitzusingen, woraufhin die Zuhörer natürlich genau dies 
taten. Der zuständige Richter des Amtsgerichts Kleve verurteilte die Mitglieder der Gruppe jeweils zu 1000 DM Geldstrafe. Offensichtlich hatte er, wie die Musiker später freimütig eingestanden, die finanzielle Situation der Gruppe zu ihren Gunsten falsch eingeschätzt (Seim 1997: 294). Im Verlauf ihrer Karriere lieferten sich Die Ärzte ein zähes Ringen mit der Bundesprüfstelle. Nachdem das Verbot der ersten beiden Platten die Band beinahe finanziell ruiniert hatte, profitierte ihr Image später von diesem subversiven »Gütesiegel «, sodass weitere Variationen des Liedes - »Claudia hat jetzt ein Pferd « und »Claudia III« (die Kernaussage war: »Claudia hat jetzt 'nen Mann und fängt ein neues Leben an, keine Sauereien mehr, nur christlicher Verkehr «) - als kalkulierte Provokationen der BPjM entstanden, die von dieser aber nicht beanstandet wurden.

Indizierungen beziehen sich immer auf einzelne Lieder bzw. Titel eines Albums, sodass die übrigen Lieder der Platte in einer anderen Zusammenstellung neu verlegt werden dürfen. Bei der Eröffnung eines Indizierungsverfahrens werden die davon betroffenen Künstler in Kenntnis gesetzt, um Einspruch einlegen und ihre Position vertreten zu können. Ein ausgesprochenes Verbot gilt 25 Jahre lang (bis zur letzten Novelle des Jugendschutzgesetzes war die Dauer einer Indizierung nicht begrenzt) und erlischt automatisch, wenn der entsprechende Titel nicht aufgrund eines neuen Verfahrens der Behörde wieder in die Liste aufgenommen wird. Zudem können Betroffene einen Antrag auf Wiederaufnahme eines Verfahrens mit dem Ziel der Listenstreichung stellen, wenn sich im Lauf der Jahre die Sach- oder Rechtslage bezüglich eines Titels geändert hat. Für die betroffenen Lieder der Ärzte traten beide Fälle ein. Einerseits wurde die Indizierung des am 27. Januar 1987 verbotenen Songs »Geschwisterliebe« am 2. Dezember 2004 bestätigt (BPjM 2004a: 3). ${ }^{7}$ Andererseits waren knapp zwei Monate zuvor im Oktober 2004 die gegen die Songs »Schlaflied« und »Claudia hat 'nen Schä-

7 In der ausführlichen sechsseitigen Begründung wurde auf die ursprüngliche Erläuterung aus dem Jahr 1987 zurückgegriffen: »Der Inhalt von Geschwisterliebe wurde als jugendgefährdend erachtet, weil der Liedtext den Straftatbestand des Beischlafs zwischen leiblichen Geschwistern nach § 173 Abs. 2 S. 2 StGB verherrliche, verharmlose und propagiere. Der Vollzug des Inzests werde als besonderer Genuss dargestellt und es bestünde die Gefahr, dass bei jugendlichen Hörern der Eindruck erweckt werde, Geschwisterliebe sei populär und vorteilhaft und es sei fortschrittlich, sich über die Verbotsbestimmung hinwegzusetzen. Das Gremium sah hierbei nicht die Gefahr in Nachahmungseffekten, sondern darin, dass Kinder und Jugendliche möglicherweise durch die Texte dazu angehalten werden, das aggressiv-sexuelle Verhalten anderer Personen zu billigen. Das Gremium ging damals ferner davon aus, dass der Tonträger nicht als Kunstwerk im Sinne von § 1 Abs. 2 GjS [Gesetz über die Verbreitung jugendgefährdender Schriften] einzustufen sei« (BPjM 2004a: 2). 
ferhund « ausgesprochenen Einschränkungen aufgehoben worden, was für den ersten Titel folgendermaßen begründet wurde:

»Die in den Texten des Schlafliedes beschriebenen Handlungen sind in erster Linie als übertrieben, aufgesetzt und unrealistisch anzusehen. Die Taten des Monsters können von heutigen Jugendlichen aufgrund deren Medienerfahrung ohne Schwierigkeit als Fiktion eingeordnet werden. Für sie ist unschwer erkennbar, dass der Text eine völlig irreale Handlung ohne jedweden Realitätsbezug schildert, so dass auch Verrohungseffekte nicht zu vermuten sind. Auch vor dem Hintergrund der damals als gewalthaltig beanstandeten Passagen ist eine jugendgefährdende Wirkung des Titels nicht anzunehmen. So erfolgt die Beschreibung der Gewalttaten des Monsters, die in nur drei der insgesamt achtzehn Textzeilen zu Tage tritt, weder detailliert, noch werden die Taten positiv bewertet. Das Lied ist aus heutiger Sicht eindeutig dem Bereich der Satire zuzuordnen und darf als solches die Kunstfreiheit des Art. 5 Abs. 3 GG für sich in Anspruch nehmen. Anders als zur damaligen Zeit ist das Gremium der Auffassung, dass sich die Aussage des Schlafliedes keinesfalls in selbstzweckhaften Gewaltbeschreibungen erschöpft, sondern als künstlerische Umsetzung kindlicher (Ur-)Ängste zu werten ist, deren künstlerischer Gehalt zumindest nicht als unbedeutend eingestuft werden kann. Hinzukommt, dass auch die einschlägigen Rezensionen den verfahrensgegenständlichen Musiktiteln durchaus einen künstlerischen Gehalt bescheinigen. So wird das Schlaflied aufgrund seiner musikalischen Umsetzung insbesondere seines Arpeggios (Spielanweisung, einen Akkord nicht mit einem Griff anzuschlagen, sondern ihn harfenartig mit nacheinander aufsteigenden Tönen zu spielen) gelobt und ihm hinsichtlich des Liedtextes bescheinigt, dass er satirisch kindliche Ängste darstelle« (BPjM 2004b: 3).

Die Sprache der Gutachten vermittelt eine große Distanz zu den bewerteten musikalischen Sachverhalten und lässt sich zweifelsfrei juristisch versierten Autoren zuordnen. Hieraus ergeben sich wichtige Hinweise, auf welcher Grundlage die Bewertungen getroffen und wie sie für eine potenzielle Überprüfung vor Gericht abgesichert wurden. Zum zweiten Lied lautete die Quintessenz der Gutachter:

»Ferner kam das Gremium auch im Hinblick auf den zweiten für die damalige Entscheidung relevanten Titel Claudia hat 'nen Schäferhund zu dem Ergebnis, dass dieser Titel auf heutige Jugendliche kaum mehr eine beeinträchtigende Wirkung ausübt. Die Textzeilen sind insbesondere nicht pornographisch, zumal der Geschlechtsverkehr zwischen Claudia und ihrem Hund zu keiner Zeit offen beschrieben wird. Die Textzeilen >Abends springt er in ihr Bett und dann geht es rund < bzw. >Am allerliebsten mag sie es, mit ihrem Hundchen unterm Esstisch erachtete das Gremium auch nicht als unsittlich, da die sodomitische Handlung zwar angedeutet wird, aber aufgrund der 
getroffenen Formulierung auch viel Raum für andere Interpretationen, auch nicht-sexueller Art, bleibt. Der sexuelle Bezug ergibt sich ausschließlich aus dem Hinweis auf Claudias >nicht-lesbische< Neigung. Hier vertrat das Gremium allerdings die Ansicht, dass der Text, auch wenn man die Zeilen sexuell interpretiere, keinesfalls Sodomie propagiere, sondern vielmehr als eine Satire auf dieses gesellschaftliche Tabu zu werten sei. Auch sei ein Nachahmungseffekt bei Kindern und Jugendlichen nicht $\mathrm{zu}$ vermuten « (BPjM 2004b: 3).

\section{HipHop - Fler und die Neue Deutsche Welle}

Bereits mit dem ersten Sampler Aggro Ansage Nr. 1 machte das Label Aggro Berlin durch besonders gewaltbereite, sexistische, homophobe, rassistische und Drogen verherrlichende Texte auf sich aufmerksam. Auch alle drei folgenden Alben Aggro Ansage Nr. 2, 3 und 4 waren Gegenstand von BPjMVerfahren und wurden aufgrund der beanstandeten Titel »Pussy« und »Psycho Neger B« (auf Ansage Nr. 2), »Call-a-Neger«, »Bums mich«, »Disziplin« und »Für die Sekte« (auf Ansage Nr. 3) sowie »Ich rappe RMX«, »Küss die Faust « und »Der Ficker « (auf Ansage Nr. 4) indiziert.

Wie in den vorangegangenen Verfahren nahmen im Verlauf des vierten Prüfungsvorgangs bevollmächtigte Juristen zur Derbheit und Aggressivität der Texte ausführlich Stellung und verwiesen auf genrespezifische Regeln, bei denen der subkulturelle Zusammenhang des HipHop zu berücksichtigen sei. Diese Ausführungen sind ein interessantes Studienmaterial, da sie all jenen die elementaren Überzeugungen der Rapper zu Musik, Kultur, Politik und Gesellschaft vermitteln sollen, die nicht mit den subkulturellen Codes und Mechanismen vertraut sind (dies mag die Länge des folgenden Zitates entschuldigen):

»Grundlegende Idee des HipHop sei der gewaltfreie Wettkampf in der Disziplin des Rap mittels einer künstlerischen Darbietung. Diese Auseinandersetzung verfeindeter Gruppen sei als >Battle< (Kampf) zu bezeichnen und mache körperliche Gewalt obsolet. Um den Gegner zu besiegen, bediene sich der Rapper bei Bildern und Metaphern aus Themenbereichen des Lebens afroamerikanischer Jugendlicher aus den US-Ghettos, der Geburtsstätte des HipHop. Der inhaltliche Bezug zu Gewalt, Sex und Drogen sei daher nicht verwunderlich, die Aussagen dürften aber nicht wörtlich genommen werden. Formulierungen wie: >Ich fick dich`, oder: >Arschficken sexuelle Handlung, sondern bedeuteten: >Ich übertreffe dich ‘. Anzumerken sei hierzu, dass diese Ausdrücke nicht nur im subkulturellen Bereich des Hip- 
Hop, sondern in der gesamten Jugend die Bedeutung >übertreffen habe. Auch Provokationen des Gegners durch Herabwürdigung von dessen Mutter oder anderen Familienmitgliedern seien keine ernst gemeinten Gewaltandrohungen. Themen wie Gewalt, Sex und Drogen gehörten zum in den US-Ghettos entstandenen HipHop traditionell dazu. Sie seien auch Inspirationsquelle für deutsche Rapper wie die Interpreten der verfahrensgegenständlichen CD, die zudem ebenfalls aus sozial schwierigen Verhältnissen stammten. Gewalt und Drogen sollten somit nicht verherrlicht, sondern kritisch beleuchtet werden. Dem Publikum sei bewusst, dass die überspitzten Gewaltandrohungen lediglich ein Substitut, ein spielerisch-metaphorischer Umgang mit physischen Gewaltanwendungen seien « (BPjM 2005: 18).$^{8}$

Jene Songs, die aufgrund explizit frauenfeindlicher sowie Gewalt verherrli-

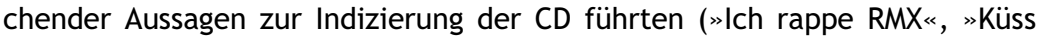
die Faust « und »Der Ficker«), sollen hier nicht thematisiert werden, da die Wirkung der Musikstücke, »Kinder und Jugendliche sozialethisch zu desorientieren «, in der 29-seitigen Entscheidungsbegründung ausführlich diskutiert wurde. Man bedachte dabei auch mögliche satirische oder kritische Absichten und ließ ferner die schwierigen Kindheiten der Musiker sowie das gewaltbereite Umfeld, in dem sie aufwuchsen, nicht unerwähnt. ${ }^{9}$ Zur Überprüfung der eingangs skizzierten Überlegungen zu musikalischen Subkulturen bietet sich vielmehr der nicht indizierte zweite Titel »Neue Deutsche Welle« von Fler auf dem vierten Aggro-Sampler an, der auf der Internetseite der Plattenfirma (www.aggroberlin.de) auch als Video heruntergeladen werden kann und in dieser Fassung auf einer sehr interessanten Vorlage basiert.

8 Das Entscheidungsgremium nahm zu dieser Begründung der sprachlichen Vulgarität Stellung: »Im Übrigen gehen die Mitglieder des 12er-Gremiums - entgegen dem Argument des Verfahrensbevollmächtigten - nicht davon aus, dass das Wort »ficken « bei sämtlichen in Deutschland lebenden Jugendlichen bereits als Synonym für >übertreffen oder sfertig machen b bekannt und etabliert ist oder dass dieses Wort im alläglichen Sprachgebrauch und in einer normalen Unterhaltung von allen Jugendlichen verwendet wird« (BPjM 2005: 25).

9 Für den Zusammenhang zu den einleitenden Überlegungen ist eine Passage des Gutachtens aufschlussreich, in der die Verantwortung und Haftbarkeit von Rappern für ihre Texte zur Sprache kommt: »Im Übrigen ist nach Auffassung der Beisitzerinnen und Beisitzer auch verbale Gewalt generell geeignet, eine verrohende Wirkung auf Kinder und Jugendliche auszuüben, gerade dann, wenn die verbale Gewalt sich so drastisch darstellt wie in diesem Liedtext [»Der Ficker «, M.C.]. Insofern sieht das 12er-Gremium das Argument der Verfahrensbeteiligten, es sei snur < verbale Gewalt gemeint, nicht als einen entlastenden Umstand an. Die Art und Weise, in der im Text der rücksichtslose Umgang mit vermeintlichen und tatsächlichen Gegnern beschrieben wird, ist nach Ansicht des Gremiums dazu geeignet, bei jugendlichen Zuhörern eine Abstumpfung gegenüber Gewalttaten und Verbalgewalt sowie eine Herabsetzung ihrer Mitleidsfähigkeit zu verursachen« (BPjM 2005: 26). 
Die gezielte verbale Provokation, die für alle Titel des Samplers charakteristisch ist, wurde in »Neue Deutsche Welle« mit nationalistischen Klischees zu einer umfassenden Negation des Konzepts einer offiziellen Leitkultur angereichert, wie sie in der Bundesrepublik seit einigen Jahren beispielsweise von Friedrich Merz und Norbert Lammert propagiert wird. ${ }^{10}$

Die von Fler eingesetzten Assoziationen funktionieren vordergründig sehr einfach, indem mit deutscher Geschichte verknüpfte Symbole (Frakturschrift, Fahne, Adler und blaue Augen) für die eigene Subkultur reklamiert werden, vermischt mit dort gültigen Werten und Erfolgsindikatoren (Geld, Frauen, Autos). Zugleich orientiert sich Flers verbale und optische Inszenierung - ein Gangster im Kreis seiner Gang, mit Goldkette und dem typischen Gehabe, verfeindete Rapper zu beschimpfen - an aktuellen US-amerikanischen Superstars wie Eminem und 50Cent. Wie hintergründig aber vom »ersten Deutschen, der richtig Welle schiebt « die Summe dieser Einflüsse eingesetzt wird, um den »deutschen Kids« zu zeigen, »wie man es macht« (wie es im Text heißt), zeigt sich erst in der Bebilderung des Stücks. ${ }^{11}$

Da nach einem Beschluss des Bundesverfassungsgerichts vom 27. November 1990 (NJW 91, 1471ff.) auch der Jugendschutz Verfassungsrang hat (abgeleitet aus Art. 1 Abs. 1, Art. 2 Abs. 2 und Art. 6 Abs. 2 GG), ist der Bundesprüfstelle aufgegeben - wie es in der schriftlichen Begründung der Sampler-Indizierung heißt - ,

»zwischen den Verfassungsgütern Kunstfreiheit und Jugendschutz abzuwägen und festzustellen, welchem der beiden Rechtsgüter im Einzelfall der Vorrang einzuräumen ist. Dabei ist bei einem Werk nicht nur die künstlerische Aussage, sondern auch die reale Wirkung zu berücksichtigen« (BPjM 2005: 27).

Die reale Wirkung von Flers Song ist, zumindest in diesem Rahmen, kaum adäquat nachzuverfolgen, wobei zu bedenken ist, dass die Indizierungen der Aggro-Sampler Nr. 2, 3 und 4 und die davon ausgelösten Kontroversen dem Ruf der davon betroffenen Musiker nicht geschadet haben, sondern im Gegenteil die Verkaufszahlen der CDs bis zu ihrem Verbot in sechsstellige

10 Im seinerzeit kontrovers diskutierten so genannten Hessischen Fragebogen, dem Leitfaden Wissen und Werte in Deutschland und Europa, dient gerade auch Musik zum Transport einer offiziellen Leitkultur, da sie den Vorteil bietet, die eigenen Vorstellungen umschreiben zu können, ohne das dahinter stehende Kulturverständnis definieren zu müssen. Ein Beispiel hierfür wäre die 83. der 100 Fragen: »Welcher Deutsche komponierte in seiner 9. Sinfonie am Schluss die berühmte >Ode an die Freude. Nennen Sie zwei weitere deutsche Musiker bzw. Komponisten! « (HmdlufS 2006).

11 Die beiden verglichenen Videos von Fler und Falco sind beim Internetportal YouTube leicht zu finden. 
Größenordnungen beförderte und den Rappern und ihrer Plattenfirma umfangreiche mediale Beachtung sicherte. In den Diskussionen über die potenzielle Wirkung ihrer Musik auf Jugendliche ziehen sich die Künstler des Aggro-Labels immer wieder auf die Freiheit der Kunstausübung zurück und verweisen auf den Realismus ihrer Texte, der Bildern aus Randbereichen einer Gesellschaft entspringe, die von der Mehrheitskultur nicht gerne wahrgenommen und gerade auch dadurch in ihrer sozialen Sprengkraft unterschätzt würden.

Ein nebengeordneter Gedanke dieser Ausführungen, in den geschilderten subkulturellen Konzeptionen das Postulat einer alternativen Leitkultur zu sehen, lässt sich auch als innermusikalischer Strang bei Fler nachverfolgen. Der Titel des Stücks bezieht sich auf einen Stil der bundesdeutschen Popmusik der 1980er Jahre, der als Neue Deutsche Welle (vielfach NDW abgekürzt) mit dadaistischen bis primitivistischen Texten und Melodien ein Gegengewicht zum damals aktuellen Hardrock und Discosound schaffen wollte. Musikalisch liegt der hier besprochenen Version der Song »Rock Me Amadeus« des 1998 verstorbenen Popsängers Falco zugrunde, der ironisch mit Klischees von Wien als Stadt der Boheme und von Mozart als Popstar spielte und sich seit der Veröffentlichung 1985 zum Evergreen entwickelte.

Vergleicht man Flers Video mit dem zwanzig Jahre älteren Clip von Falco, so sind einige adaptierte und erkennbar variierte Motive offensichtlich: das Betreten und die Inbesitznahme von Räumen (Straßen, Hinterhöfe und urbane Wüsten in Berlin versus Wiener Palais), Fahrzeuge als Statussymbole (Benzin- und Pferdekutschen), das Zurschaustellen von Macht (eine besprühte S-Bahn, Kampfhunde, Polizeirazzias und Faustrecht beim einen, Adel und Klerus nebst einer Rockerbande beim anderen), der Outlaw Fler im Kreise seiner Vertrauten sowie der Künstler Mozart als Pop- und Medienstar inmitten seiner Fans, begeisterte, bei Fler offensichtlich zu Huren degradierte Frauen neben harten, starken Männern (bei Falco in Person der Rocker). Allein die Austragungsform von Konflikten stellt einen wesentlichen Unterschied in den Aussagen der beiden Videos dar: die verbrüdernde Wirkung der Musik eines modernen Mozarts bietet einen Gegenentwurf zu Bandenrivalitäten und Kampfsequenzen im Stil der Szene-Kultfilme Blood Sport (1986) oder Fight Club (1999) bei Fler.

Wenn Fler einen Hit des verehrten Vorbilds Falco - dessen Konterfei er im Video als T-Shirt trägt - mit einem neuen Text versieht und selbstreferenziell die eigene, plötzliche Präsenz im Musikfernsehen VIVA kommentiert, ist dies zugleich der Versuch, seinen anderen großen, amerikanischen Vorbildern nachzueifern, die als ehemalige Ghettokids mit geschicktem Marketing, eigenen Modelinien und Plattenfirmen den amerikanischen 
Traum von Ruhm und Reichtum verkörpern und HipHop und Rap zu einer wichtigen Sparte des globalen Musikmarktes ausbauten.

Wie die im Vergleich sehr unterschiedlichen Beispiele von Punk und HipHop deutlich machten, beginnen unter dem Blickwinkel von Indizierung und Selbstinszenierung die Grenzen zwischen Subkultur und Mainstream, zwischen Mehrheitsnormen und Minderheitsmeinungen zu changieren, je nach Standpunkt und Attitüde. Die Indizierung von Musik als Übertragung von absichtlich außer-künstlerischen Maßstäben auf Phänomene, denen Kunstrang eingeräumt wird, erweist sich dabei als besonders wirkungsmächtige Überschneidung von Ästhetik und ethischem Urteil. Und gerade aus den Grenzlinien zwischen Kunst und Gesetz bezieht diese Thematik ihre Spannung. Zwar ist die Wirkung der Musik nicht vollständig objektiv zu beurteilen und daher umso strittiger. Die Wirkung der Urteile über Musik ist aber deutlich und betrifft alle beteiligten Instanzen: Behörden und Institutionen bemühen sich um die Aufrechterhaltung von Rechtsstaatlichkeit, die Musiker profitieren von der eigenen Mystifizierung und der indirekten Werbung für ihre Tonträger. Gesellschaftlich zeigt sich ein wandelndes, zeitbezogenes Gefühl für Zensur und Satire.

\section{Literatur}

Bundesprüfstelle für jugendgefährdende Medien (BPjM) (2004a). Entscheidung Nr. A 104/04 vom 29.10.2004. Aktenzeichen Pr. 607/04.

Bundesprüfstelle für jugendgefährdende Medien (BPjM) (2004b). Entscheidung Nr. 5264 vom 02.12.2004. Aktenzeichen 605/04.

Bundesprüfstelle für jugendgefährdende Medien (BPjM) (2005). Entscheidung Nr. 5313 vom 1.9.2005. Aktenzeichen Pr. 200/05.

Bundesprüfstelle für jugendgefährdende Medien (BPjM) (2008). »Indizierungsverfahren / Verfahrensarten / Verfahren vor dem 12er-Gremium. " Online unter: http: //www.bundespruefstelle.de/bmfsfj/generator/bpjm/Jugendmedienschutz/Indi zierungsverfahren/verfahrensarten,did=32934.html (Zugriff: 19.5.2008).

Carus, Birgit / Hannak-Mayer, Martina / Kortländer, Ute (2006). »Hip-Hop-Musik in der Spruchpraxis der Bundesprüfstelle. "Sonderdruck aus: BPjM aktuell. Amtliches Mitteilungsblatt der Bundesprüfstelle für jugendgefährdende Medien. H. 1. Online unter: http://www.bundespruefstelle.de/bmfsfj/generator/bpjm/ redaktion/PDF-Anlagen/bpjm-aktuell-sonderheft2006-hip-hop-musik, property= pdf, bereich=bpjm, sprache=de,rwb=true.pdf (Zugriff: 15.3.2008).

Chastagner, Claude (1999). "The Parents' Music Resource Center: From Information to Censorship.«In: Popular Music 18, H. 2, S. 179-192.

Gracyk, Theodore (1996). Rhythm and Noise. An Aesthetics of Rock. Durham, London: Duke University Press.

Hessisches Ministerium des Innern und für Sport (HmdlufS) (Hg.) (2005). Leitfaden Wissen und Werte in Deutschland und Europa. Online unter: http://www. 
einbuergern.de/pdf-ordner/stag/leitfaden_wissen_werte_indt-u-europa.pdf (Zugriff: 15.3 .2008$)$.

Miehling, Klaus (2006). Gewaltmusik. Musikgewalt. Populäre Musik und die Folgen. Würzburg: Königshausen und Neumann.

Müller, Kai / Wunder, Jörg (2005). „Schlimme Wörter. Innenminister Sarkozy will Hip-Hop-Musiker wegen Volksverhetzung ins Gefängnis bringen. Ein Kulturkampf.« In: Der Tagesspiegel vom 6. Dezember. Online unter: http://www. tagesspiegel.de/kultur/;art772,1964191 (Zugriff: 15.3.2008).

Seim, Roland (1997). Zwischen Medienfreiheit und Zensureingriffen. Eine medienund rechtssoziologische Untersuchung zensorischer Einflußnahmen auf bundesdeutsche Populärkultur. Münster: Telos.

\begin{abstract}
Music serves as an important means for adolescents to position themselves within their social surrounding and to show distance to values and traditions of adults. The border between child-welfare and artistic freedom in Germany is observed by the „Bundesprüfstelle für jugendgefährdende Medien « (Federal Department for Media Harmful to Young Persons) whose decisions often cause controversial discussions. This essay is based upon reports of indexing proceedings of the agency, which usually are not published. They can give an instructive insight into normative ways of thinking about music and youth culture which are normally hardly accessible but socially and cultural politically highly effective.
\end{abstract}

